\title{
An algorithm for the investigation and management of patients with suspected deep venous thrombosis at a district general hospital
}

\author{
K Walsh, N Kelaher, K Long, P Cervi
}

Postgrad Med J 2002;78:742-745

See end of article for authors' affiliations

Correspondence to:

Dr Kieran Walsh, Basildon

Hospital, Nether Mayne,

Basildon, Essex SS16 5NL,

UK; kieran@

kmwalsh.freeserve.co.uk

Submitted 27 May 2002

Accepted

8 October 2002

\begin{abstract}
The aim was to show that clinical assessment and serial Dopplers could be used to exclude deep venous thrombosis (DVT), and also that clinical assessment in combination with D-dimers could be used to exclude DVT. This was a prospective trial. Patients underwent clinical assessment, colour flow Doppler, and D-dimer measurement. Patients with an equivocal first Doppler and patients with a moderate/high pre-test clinical score had a repeat Doppler. Follow up information was obtained to see if further thromboembolism developed. Outcome measures included the reliability of the clinical assessment, Doppler, and D-dimer in excluding DVT. One hundred and ninety four patients were enrolled. The negative predictive value of a low medical pre-test score in excluding DVT was $92 \%$. Eighty eight patients underwent $\mathrm{D}$-dimer measurement. The negative predictive value of a $\mathrm{D}$-dimer $<400$ units was $96 \%$. The negative predictive value of a low clinical pre-test score and D-dimer $<400$ units was $100 \%$. The negative predictive value of a low clinical pre-test score and D-dimer $<800$ units was $97 \%$. The negative predictive value of the combination of a low clinical pre-test score and D-dimer $<400$ units was $100 \%$. Twenty three per cent of patients had a low clinical pre-test score and D-dimer $<400$ units. Thus, a DVT could be excluded in $23 \%$ of referred patients. This information could be used to reduce Doppler requests by $23 \%$. No patient in whom clinical assessment and serial Dopplers had excluded a DVT suffered further thromboembolism on follow up. This study has thus shown that a low medical pre-test score and negative Doppler reliably exclude DVT; a low pre-test score and negative D-dimer would reliably exclude DVT.
\end{abstract}

eep venous thrombosis (DVT) and pulmonary embolism are common causes of morbidity and mortality. There is increasing concern at the inconsistent approach to the investigation of suspected DVT. The traditional view is that clinical assessment of suspected DVT is unreliable and that diagnostic techniques such as venography are invasive and expensive. This has lead to general reliance on Doppler ultrasound to confirm or refute the diagnosis. Clinical pre-test assessment of suspected DVT can guide subsequent radiological investigation; this allows a more rational use of radiological resources. ${ }^{2}$ Compression ultrasonography is widely accepted as the best non-invasive test in the diagnostic assessment of patients with suspected DVT. ${ }^{3}$ Colour flow Doppler is rapidly becoming the de facto gold standard radiological investigation; its routine availability in UK district hospitals is becoming the norm. Labropoulos et al and Amin et al have shown that colour flow Doppler is as accurate as venography in the diagnosis of DVT. ${ }^{4}$

Compression ultrasonography is an accurate test in the diagnosis of DVT in symptomatic patients. ${ }^{6}$ However, if the initial results of the ultrasound are normal in patients with a suspected DVT, clinicians should withhold anticoagulants and perform repeat ultrasonography after seven to 14 days. Although this approach is safe and effective, it is also inconvenient and expensive. A complementary test that, in combination with a low clinical probability, could exclude the presence of thrombi would obviate the need for ultrasonography in some patients. D-dimer is a plasmin derived degradation product of cross linked fibrin. D-dimer assays have high sensitivity but low specificity in the diagnosis of DVT. ${ }^{78}$ When the results of a D-dimer assay and impedance plethysmography examination are normal, deep venous thrombosis is excluded. ${ }^{10}$
As it is not acceptable to miss a DVT in any patient, the negative predictive value of any test (or combination of tests) must approach $100 \%$. In this study, we evaluated the usefulness of clinical evaluation and serial colour flow Doppler in the diagnosis of DVT. We also analysed D-dimer concentrations in the patients to establish a normal cut off level in our laboratory. There are different ways of assessing D-dimer; each method must be validated locally. We investigated whether clinical evaluation in combination with the D-dimers could exclude the presence of DVT. We followed up the study patients for six months after completion of anticoagulation to confirm the safety of our investigative algorithm. We obtained ethical committee approval from out local research ethics committee before proceeding with the study.

\section{SUBJECTS AND METHODS \\ Participants}

This was a prospective cohort trial. Consecutive patients referred to casualty and the general practitioner referral unit of Basildon Hospital, Essex, England with a suspected DVT constituted the study population. Recruitment began in September 1998 and ended in February 2000 and follow up completed in December 2000.

We excluded patients from the study for any of the following reasons: previous history of radiologically proved DVT in the affected limb, clinical features of current pulmonary embolism or past history of pulmonary embolism, life expectancy less than six months, age less than 16 years, requirement for long term anticoagulation, non-availability for follow up, and failure or inability to give informed consent. Inpatients were excluded from the study. The local ethics research committee approved the study protocol. 
Table 1 Simplified pre-test clinical score

\begin{tabular}{ll}
\hline Clinical feature & Score \\
\hline $\begin{array}{l}\text { Active cancer (treatment ongoing or within the previous six } \\
\text { months) }\end{array}$ & 1 \\
Paralysis, paresis or recent plaster immobilisation of lower & 1 \\
limbs & 1 \\
$\begin{array}{l}\text { Recently bed ridden for more than three days or major } \\
\text { surgery within four weeks }\end{array}$ & 1 \\
$\begin{array}{l}\text { Localised tenderness along the distribution of the deep } \\
\text { venous system }\end{array}$ & 1 \\
$\begin{array}{l}\text { Entire leg swollen } \\
\text { Calf swelling by more than } 3 \text { cm when compared to the } \\
\text { asymptomatic leg* }\end{array}$ & 1 \\
$\begin{array}{l}\text { Pitting oedema (greater in the symptomatic leg) } \\
\text { Collateral superficial veins (non-varicose) }\end{array}$ & 1 \\
Alternative diagnosis as likely or greater than that of DVT & -2 \\
\hline *Measured $10 \mathrm{~cm}$ below the tibial tuberosity. & 1
\end{tabular}

\section{Clinical evaluation}

Anticoagulation nurse practitioners or junior doctors evaluated the patients. They used a pre-test clinical probability assessment devised by Wells et $a^{2}$ (table 1). In their study, patients with a score of 3 or more are deemed "high probability" and have a $75 \%$ chance of DVT; patients with a score of 1 or 2 are deemed "moderate probability" and have a $16.6 \%$ chance of DVT; low probability patients with a score of zero or less have a $3 \%$ chance of DVT. The ultrasonographer subsequently repeated this pre-test clinical assessment before performing the Doppler examination. Each assessor was blind to the other's assessment. We then managed the patients according to the algorithms outlined in figs 1 and 2 .

\section{Colour flow Doppler ultrasonography}

Radiographers in the ultrasonography department performed the Dopplers. The radiographers at our hospital have considerable experience in performing colour flow Doppler ultrasonography (including scanning of the calf veins). Our ultrasonographers have routinely scanned all the calf veins in all patients with a suspected DVT since 1993.

The results were interpreted as follows: (1) normal, if all deep veins (including the calf veins) were adequately visualised and normal; (2) diagnosis of DVT, if an intraluminal defect was present; (3) equivocal, all other patterns. The following patients had a second Doppler scan two to four days after the first scan:

- Patients who had an equivocal first Doppler.

- Patients who had a moderate/high pre-test score and a normal first Doppler.

All patients with a confirmed DVT (including patients with a single calf vein thrombus) received anticoagulation.

\section{Venography}

Patients with an equivocal result on the second Doppler had a venogram performed by a radiologist.

\section{Laboratory investigation}

The anticoagulation nurse practitioner or junior doctor collected blood from the antecubital vein. We carried out the

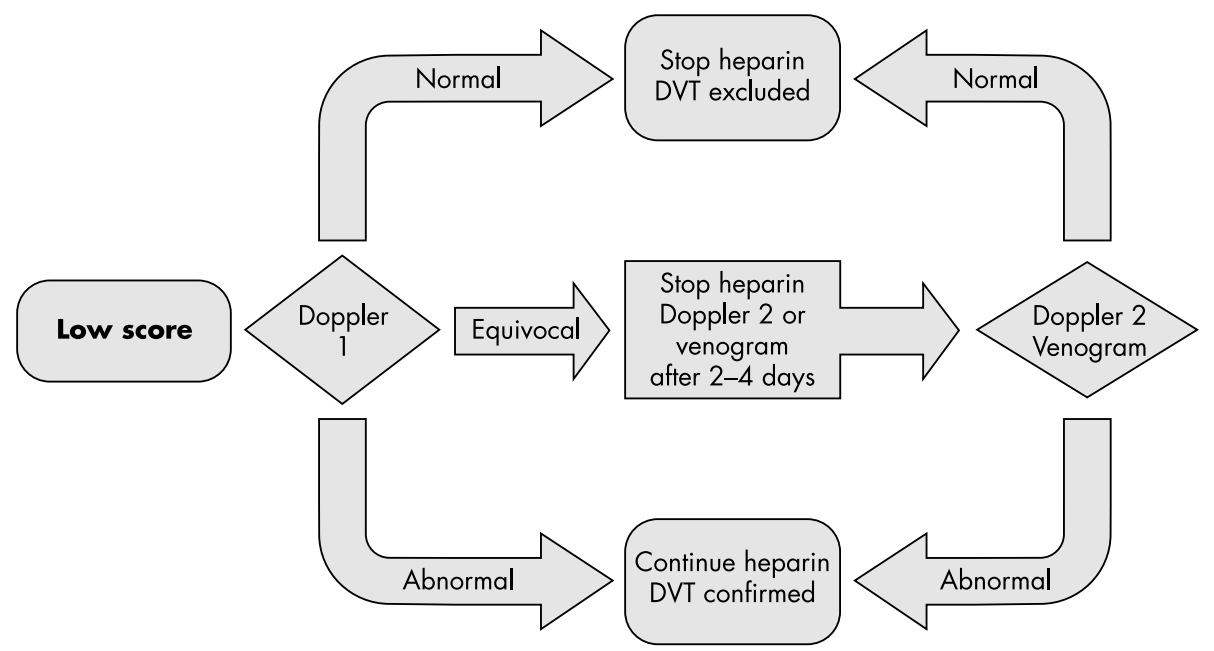

Figure 1 Low pre-test score.

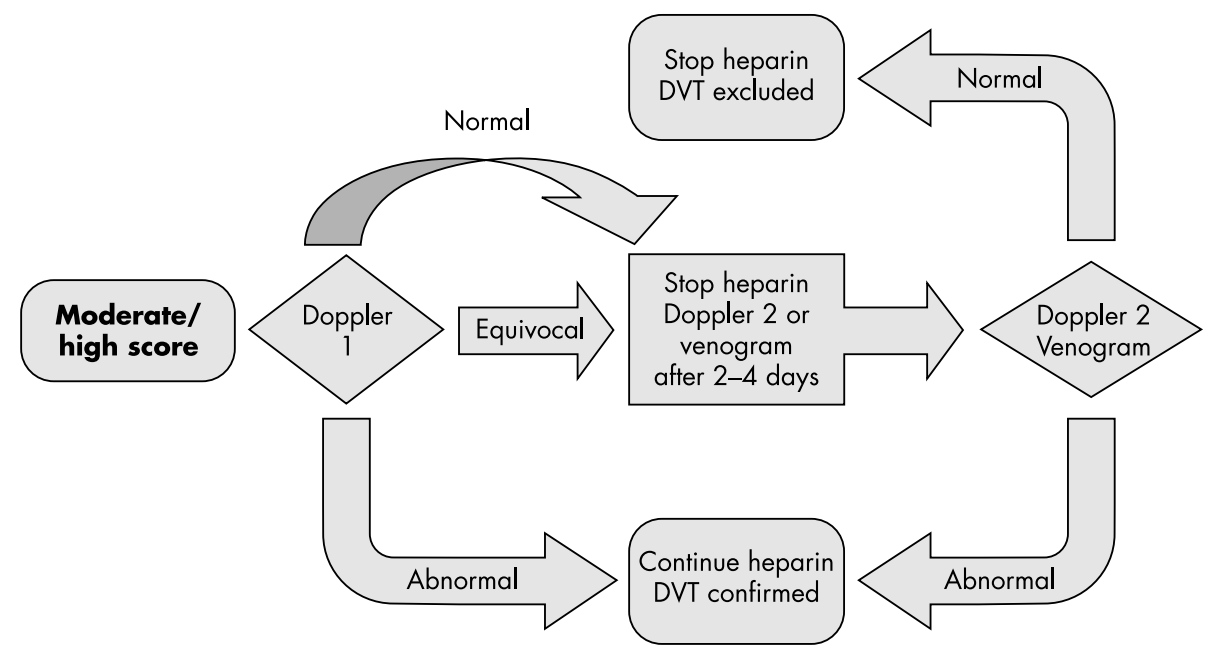

Figure 2 Moderate or high pre-test score. 


\begin{tabular}{|ll|}
\hline Table 2 & Reasons for exclusion \\
\hline Reason for exclusion & $\begin{array}{l}\text { No of } \\
\text { patients }\end{array}$ \\
\hline Terminal cancer & 14 \\
Possible pulmonary embolism & 9 \\
Definite pulmonary embolism & 2 \\
Confusional state & 12 \\
Already started on heparin & 7 \\
Already on long term & 4 \\
anticoagulation & 1 \\
Recent cerebrovascular accident & 13 \\
Previous DVT & 43 \\
\hline
\end{tabular}

Vidas D-dimer assay in the laboratory. Although we did not use the results in this study to guide further investigation or management, this approach allowed us to evaluate the need for Doppler examinations in future practice.

\section{Questionnaires}

Six months after completion of anticoagulation, we sent a questionnaire to the patients. We asked if symptoms or signs of DVT or pulmonary embolism had developed during the interval. We sent a follow up letter to the general practitioners of those patients who did not return the questionnaire and to those in whom we had diagnosed venous thrombosis.

\section{Data and statistical analysis}

We calculated the negative and positive predictive values of the clinical assessment and D- dimer assay (both alone and in combination).

\section{RESULTS}

During the course of the study, 304 patients attended the centre with a suspected DVT. Eighteen refused consent to participate. We excluded 92 patients for the reasons listed in table 2 . Thus, we enrolled 194 patients into the study. Their mean (SD) age was 57.6 (18.7) years. There were 52 males and 142 females.

\section{Medical pre-test scores}

One hundred and ninety one patients had a medical pre-test score. An anticoagulation nurse practitioner performed the medical pre-test score in $82 \%$ of the patients and a doctor in $18 \%$ of the patients. One hundred and seven patients (55\%) had a low score and 84 patients (45\%) had a medium/high score.

\section{Ultrasonography pre-test scores}

The ultrasonographer performed the ultrasonography pre-test score in 184 patients. Ninety nine patients $(54 \%)$ had a low score and 85 patients (46\%) had a medium/high score.

\section{Result of D-dimer analysis}

A D-dimer analysis was performed in 88 patients. The accuracy indices of a D-dimer $<400$ units are outlined in table 3.

\section{Predictive value of the combination of a low medical pre-test score and negative D- dimer with a cut off for $\mathrm{D}$-dimer between $\mathbf{4 0 0}$ and $\mathbf{8 0 0}$ units}

The accuracy indices of the combination of a low medical pretest score and a negative D-dimer (at differing levels for a negative D-dimer result, between 400 and 800 units) are outlined in table 4.

\section{Percentage of patients (where a D-dimer analysis was} performed) who had a low medical pre-test score at differing levels of $D$-dimer cut off

Twenty three per cent of patients had a low medical pre-test score and a D-dimer $<400$ units. Thirty four per cent of patients had a low medical pre-test score and a D-dimer $<800$ units.

\section{Result of first Doppler}

The mean wait between entering the study and having the first Doppler was 0.97 days. The results of the first Doppler are outlined in table 5 .

\begin{tabular}{|lllll|}
\hline Table 3 & Result of D-dimer analysis \\
\hline & $\begin{array}{l}\text { DVT present } \\
\text { (confirmed by } \\
\text { Doppler) }\end{array}$ & DVT absent & $\begin{array}{l}\text { Positive predictive } \\
\text { value (\%) }\end{array}$ & $\begin{array}{l}\text { Negative } \\
\text { predictive value } \\
\text { (Cl) (\%) }\end{array}$ \\
\hline $\begin{array}{l}\text { Positive }(>400 \mathrm{U}) \\
\text { Negative }(<400 \mathrm{U})\end{array}$ & 17 & 46 & 27 & - \\
\hline $\mathrm{Cl}$, confidence interval. & 1 & 24 & - & 96 (87 to 106) \\
\hline
\end{tabular}

\begin{tabular}{|c|c|c|c|c|}
\hline Combination & $\begin{array}{l}\text { DVT present } \\
\text { (confirmed by } \\
\text { Doppler) }\end{array}$ & DVT absent & $\begin{array}{l}\text { Positive predictive } \\
\text { value (\%) }\end{array}$ & $\begin{array}{l}\text { Negative } \\
\text { predictive value } \\
\text { (CI) (\%) }\end{array}$ \\
\hline \multicolumn{5}{|c|}{ Low score + D-dimer (units) } \\
\hline$>400$ & 4 & 25 & 14 & \\
\hline$<400$ & 0 & 20 & - & 100 (98 to 103$)$ \\
\hline$>500$ & 4 & 23 & 15 & \\
\hline$<500$ & 0 & 22 & - & 100 (98 to 102$)$ \\
\hline$>600$ & 4 & 19 & 17 & \\
\hline$<600$ & 0 & 26 & - & 100 (98 to 102$)$ \\
\hline$>700$ & 3 & 16 & 16 & \\
\hline$<700$ & 1 & 29 & - & 97 (92 to 102$)$ \\
\hline$>800$ & 3 & 15 & 17 & \\
\hline$<800$ & 1 & 30 & - & 97 (92 to 102 ) \\
\hline
\end{tabular}




\begin{tabular}{ll|}
\hline $\begin{array}{l}\text { Table } 5 \\
\text { Dopplers }\end{array}$ & Results of first and second \\
\hline & No of patients \\
\hline Result of first Doppler & 129 \\
Normal & 21 \\
Equivocal & 39 \\
DVT present & 1 \\
Haematoma & 4 \\
"Not done" & \\
Result of second Doppler & 22 \\
Normal & 5 \\
Equivocal & \\
\hline
\end{tabular}

\section{Result of second Doppler}

Twenty seven patients had a second Doppler. The mean wait between first and second Dopplers was 4.8 days. The results of the second Doppler are shown in table 5.

\section{Result of venogram}

One patient with an equivocal result on both Dopplers had a normal venogram. In another such case, the venogram was impossible to perform.

\section{Result of questionnaires}

All patients were sent a questionnaire six months after completion of anticoagulation to see if symptoms or signs of DVT or pulmonary embolism had developed during the interval. We sent a follow up letter to the general practitioners of those patients who did not return the questionnaire and to those in whom we had diagnosed venous thrombosis. Sixteen patients replied that they wished to withdraw from the study without explanation. Follow up information was obtained on all the remaining 178 patients. We withheld anticoagulation from those patients in whom our investigative algorithm had ruled out a DVT. None of these patients suffered thromboembolic events during the follow up period. We anticoagulated those patients in whom we had diagnosed a DVT by our algorithm. None of these patients had a pulmonary embolism during the follow up period. One patient with DVT died from bronchopneumonia due to metastatic prostate cancer.

\section{DISCUSSION}

As it is not acceptable for a DVT to be missed in any patient, the negative predictive value of any test (or combination of tests) must approach $100 \%$. This study has shown that the negative predictive value of a low clinical pre-test score alone is $92 \%$. The negative predictive value of a normal D-dimer alone (at a cut off for normal $<400$ units) is $96 \%$. The negative predictive value of the combination of a low clinical pre-test score and D-dimer $<400$ units is $100 \%$. Twenty three per cent of patients had a low clinical pre-test score and D-dimer $<400$ units. Thus, we could exclude a DVT in $23 \%$ of referred patients. This information could be used to reduce Doppler requests by $23 \%$. The negative predictive value of the combination of a low clinical pre-test score and D-dimer $<800$ units is $9 \%$. Thirty four per cent of patients had a low clinical pre-test score and D-dimer $<800$ units. Thus, we could exclude a DVT in $34 \%$ of referred patients and thus reduce Doppler requests by $34 \%$. We believe that a strategy using the clinical pre-test score in combination with D-dimer assay could significantly reduce the need for Doppler examination.

None of the study patients in whom clinical assessment and serial colour flow ultrasonography excluded a DVT developed further thromboembolism during the follow up period. Only
27 of the 194 patients had to have a repeat colour flow ultrasound. This represents $14 \%$ of the patients. We feel that it is worthwhile rescanning $14 \%$ of the patients to ensure patient safety. The fact that no patient in whom our investigative algorithm excluded a DVT developed further thromboembolism during follow up confirms the safety of our algorithm.

Therefore, D-dimers should be part of the repertoire of laboratory tests available to the doctor on-call. Introducing this test on an on-call basis would add to the laboratory cost. However, the additional cost would be more than compensated by the reduction in the numbers of Dopplers performed and the reduction in the number of patients who require low molecular weight heparin while awaiting their Doppler. In addition, patients would not have to undergo the worry, inconvenience, and risk of daily injections while awaiting confirmation or rejection of the diagnosis. Furthermore, our study included comprehensive follow up questionnaires to the patients and their general practitioners. The overall response rate to the questionnaires was $92 \%$. None of the respondents suffered a pulmonary embolism. In conclusion, this study has shown that developments in the diagnosis of DVT (including pre-test score, colour flow Doppler ultrasonography, and D-dimer assay) can be introduced reliably and safely in a typical district general hospital in the UK. The study protocol will now be integrated into the hospital strategy for the radiological investigation of DVT. This protocol allows the diagnosis of DVT to be excluded at presentation in a significant number of patients-thus obviating the need for heparin injections and/or admission to hospital. Scarce radiological resources can now be concentrated on the high risk patients. We encourage other district general hospitals to adopt our investigative algorithm.

\section{ACKNOWLEDGEMENTS}

Many thanks to Basildon \& Thurrock NHS Hospitals Trust and South Essex Medical Education and Research Trust who gave financial support to the study.

\section{Authors' affiliations}

K Walsh, N Kelaher, K Long, P Cervi, Basildon \& Thurrock NHS Hospitals Trust, Basildon, Essex

\section{REFERENCES}

1 Wheeler HB, Anderson FA. Diagnostic approaches for deep vein thromboses. Chest 1986:89:407s-12s.

2 Wells PS, Anderson DR, Bormanis J, et al. Value of assessment of pretest probability of deep venous thrombosis in clinical management. Lancet 1997;350:1795-8.

3 Cogo A, Lensing AWA, Koopman MMW, et al. Compression ultrasonography for diagnostic management of patients with clinically suspected deep venous thrombosis: prospective cohort study. BM 1998;316:17-20

4 Labropoulos $\mathbf{N}$, Leon $\mathrm{M}$, Kalodiki $\mathrm{E}$, et al. Colour flow duplex scanning in suspected acute deep vein thrombosis; experience with routine use. Eur $J$ Vasc Endovasc Surg 1995;9:49-52.

5 Amin MA, Khan MZ, Khan MA, et al. Diagnosis of deep vein thrombosis in the leg by using colour coded duplex sonography. J Ayub Med Coll Abbottabad 2001;13:22-3.

6 Heiiboer $\mathbf{H}$, Buller HR, Lensing AWA, et al. A comparison of real time compression ultrasonography with impedance plethysmography for suspected deep venous thrombosis in outpatients. N Engl J Med 1993;329:1365-69.

7 D'Angelo A, D'Allessanmdro G, Tomassini L, et al. Evaluation of a new rapid quantitative $D$-dimer assay in patients with clinically suspected deep venous thrombosis. Thromb Haemost 1996;75:412-6.

8 Jannsen $\mathbf{M C H}$, Heebels $A E$, deMetz $M$, et al. Reliability of five rapid D-dimer assays compared to ELISA in the exclusion of deep venous thrombosis. Thromb Haemost 1997;77:602-3.

9 Wells PS, Brill-Edwards P, Stevens P, et al. A novel and rapid whole blood assay for D-DIMER in patients with clinically suspected deep vein thrombosis. Circulation 1995;91:2184-7.

10 Ginsberg JS, Kearon C, Douketis J, et al. The use of D-dimer testing and impedance plethysmographic examination in patients with clinical indications of deep vein thrombosis. Arch Intern Med 1997;157:107781 